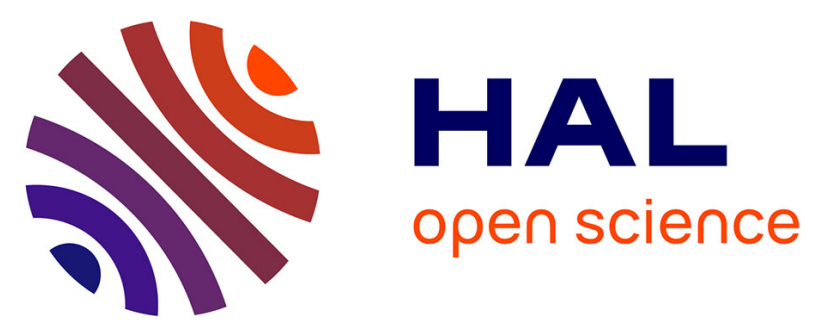

\title{
Coupled Time Resolved and High Frequency Modulated Photoluminescence probing surface passivation of highly doped ntype InP samples
}

Wei Zhao, Baptiste Bérenguier, Cendra Patie Rakotoarimanana, Anne-Marie Goncalves, Arnaud Etcheberry, Mathieu Frégnaux, L Lombez, Jean-François Guillemoles

\section{To cite this version:}

Wei Zhao, Baptiste Bérenguier, Cendra Patie Rakotoarimanana, Anne-Marie Goncalves, Arnaud Etcheberry, et al.. Coupled Time Resolved and High Frequency Modulated Photoluminescence probing surface passivation of highly doped ntype InP samples. Journal of Applied Physics, inPress, 129 (21), pp.215305. 10.1063/5.0033122 . hal-03244613

\section{HAL Id: hal-03244613 https://hal.science/hal-03244613}

Submitted on 1 Jun 2021

HAL is a multi-disciplinary open access archive for the deposit and dissemination of scientific research documents, whether they are published or not. The documents may come from teaching and research institutions in France or abroad, or from public or private research centers.
L'archive ouverte pluridisciplinaire HAL, est destinée au dépôt et à la diffusion de documents scientifiques de niveau recherche, publiés ou non, émanant des établissements d'enseignement et de recherche français ou étrangers, des laboratoires publics ou privés. 


\title{
Coupled Time Resolved and High Frequency Modulated Photoluminescence probing surface passivation of highly doped n- type InP samples
}

Wei Zhao (1), Baptiste Bérenguier (2), Cendra Rakotoarimanana (3), Anne Marie Goncalves (3), Arnaud Etcheberry (3), Mathieu Frégnaux (3), Laurent Lombez (2), Jean-François Guillemoles (2),

1) Institut Photovoltaïque d'Ile de France, 91120 Palaiseau France

2) UMR IPVF 9006, CNRS, Ecole Polytechnique Institut Polytechnique de Paris, PSL Chimie ParisTech, IPVF SAS, 91120 PALAISEAU, France

3) ILV - Institut Lavoisier de Versailles UMR 8180 CNRS/UVSQ, Saint-Quentin-en-Yvelines

\begin{abstract}
Electronic passivation of III-V surfaces is essential for applications in optoelectronic devices. A key aspect is the measurement of the surface recombination properties which can be done by various techniques including transient photoluminescence (TRPL) or luminescence quantum yield. These measurement techniques are always indirect since they suppose postulating a mathematical model for the data interpretation. Most common models use the notion of surface recombination velocity to quantify the surface recombination. In this paper we demonstrate on InP substrate than this notion is not always sufficient to represent the injection dependence of the surface phenomena. The study of power dependence of TRPL decays coupled with Modulated Photoluminescence (MPL) spectra on four samples from the same wafer with different surface treatments (epi-ready, freshly cleaned, after air exposure and with poly-phosphazen passivation) allows us for discriminating between bulk and surface properties. We introduce surface defect trapping as an alternative to explain TRPL decays and MPL phase excitation power dependences of the three non-passivated samples. Surface trap parameters such as capture cross section and defect density are extracted. The passivated sample exhibits invariant response shape on eight orders of magnitude of illumination. The stability of the PL response at high flux is in agreement with the perfect stability of the passivation layer which is able to protect the InP surface without chemical changes over more than one year. They are linked to the nature of the Passivation layer/InP interface. Other surface treatments were found to have an injection dependent response
\end{abstract}


at high flux corresponding to different surface defects distribution but also possibly to surface chemical changes for the freshly cleaned sample.

\section{INTRODUCTION}

III-V semiconductors are recognized as high-performance materials for the fabrication of (opto)electronic devices such as avalanche photodiodes, light emitting diodes and lasers. For many years, Indium Phosphide (InP) based thin film III-V emitters $\left(\mathrm{Ga}_{\mathrm{x}} \operatorname{In}_{1-\mathrm{x}} \mathrm{P}\right.$ and $\left.\mathrm{Ga}_{\mathrm{x}} \operatorname{In}_{1-\mathrm{x}} \mathrm{As} \mathrm{s}_{\mathrm{y}} \mathrm{P}_{1-\mathrm{y}}\right)$ have been commonly used by the optoelectronics industries ${ }^{1}$ and are top candidates for Photonic Integrated Components ${ }^{2}$. However, the surface and interface properties of these materials can degrade the overall performances of the layers/devices. To address this, numerous chemical treatments have been developed to improve the surface properties of InP, and first of all surface chemical and electronic passivation ${ }^{3-5}$. In parallel, advanced contactless optical characterization methods have proved to be powerful to determine carriers' dynamics and recombination mechanisms in semiconductor materials. A standard method, Time Resolved Photoluminescence (TRPL), has been used for probing sub nanosecond recombination mechanisms. Nevertheless, the interpretation of the TRPL temporal decays may be difficult when presenting non monoexponential behaviour ${ }^{6}$. Generally, determination of the surface/interface recombination mechanisms is not a simple task since there is no straightforward contactless characterization technique to measure the surface state density and other physical properties with sufficient accuracy. The main difficulty lies in the dissociation of the relative contribution of bulk and surface from the raw TRPL decays. The surface recombination velocity (SRV) can affect the TRPL decay times by causing surface depletion (or accumulation) of excess carriers and competing with bulk recombination and surface band bending ${ }^{7,8}$. The SRV in InP is supposed to increase with the doping density until it is limited by the thermal velocity of carriers. In one early study, Bothra et al. ${ }^{9}$ found that an increase of doping from $3 \times 10^{15} \mathrm{~cm}^{-3}$ to $3 \times 10^{18} \mathrm{~cm}^{-3}$ caused an increase of SRV from $5000 \mathrm{~cm} / \mathrm{s}$ to $1 \times 10^{6} \mathrm{~cm} / \mathrm{s}$. Other benchmarking studies using in situ photoluminescence (PL) quantum yield measurements or time resolved photo-gratings investigate InP surface states (attractive traps) and found fixed positions of the Fermi level at the surface and constant SRV for various doping densities of $\mathrm{InP}$ as well as several treatments of the surface ${ }^{10-12}$. Improvement of the PL yield technique was brought while using the Unified Disorder Induced Gap State model 
(DIGS) of Hasegawa postulating U-shape density of interface states ${ }^{13-15}$. Pinning of the Fermi level at about $1 \mathrm{eV}$ above the valence band maximum was observed in both highly doped $\left(10^{18} \mathrm{~cm}^{-}\right.$ $\left.{ }^{3}\right) \mathrm{n}$ and $\mathrm{p}$ type (100) InP surface despite the surface treatments. Rosenwarks et al. also studied TRPL decays on one highly $n$ doped sample but without mentioning SRV ${ }^{16}$. The authors concluded that the non-radiative recombination and the effective lifetime are insensitive to the injection level. Moreover, for undoped InP, the radiative coefficient is found to be $2 \times 10^{-11} \mathrm{~cm}^{3} / \mathrm{s}$, which is the lowest value reported. The question of the value of this coefficient for high doping remain open.

In order to provide complementary information, another approach, called Modulated Photoluminescence (MPL) or Frequency Domain Photoluminescence, can also be used to investigate carrier lifetime in semiconductors. By using sinusoidal illumination variation and recording the phase shift between the excitation signal and emitted signal, one can assess the carrier lifetime for example in silicon wafers ${ }^{17}$. Extended to the MHz range, the High-Frequency MPL (HF-MPL) can probe short lifetime and be closer to the real excitation conditions (i.e. close to continuous wave excitation by tuning the modulation amplitude). It also enables focusing on mechanisms that usually appear in the noisy end of the decay such as the carrier detrapping ${ }^{18}$. Great efforts have already been done previously on silicon and CIGS samples ${ }^{18-20}$. Reklaitis et al. also highlighted this frequency-domain photoluminescence technique in their study of carrier lifetime in InGaN light-emitting diode and proposed Fourier transform of TRPL decay models to fit the data ${ }^{21,22}$. Pulsed and modulated excitation can thus be compared if the solution for the sinusoidal excitation is derived from impulse response i.e, when the underlying mathematical equation system is linear. The proven advantage of Frequency Domain techniques is that they can investigate several orders of injection level below TRPL ${ }^{22}$.

We here adopted the approach of combined TRPL and MPL techniques to probe four highly n-type doped InP from the same wafer with different surface treatments (epi-ready, freshly cleaned, after air exposure and with poly-phosphazen passivation). Such poly-phosphazen layers have proven to protect chemically the surface since no oxidation of the initial clean surface was observed over more than 1 year ${ }^{23-25}$. Detailed XPS analysis can be found in Gonçalves et al ${ }^{26}$., It is yet to be investigated how they passivate the surface electronically and behave at high illumination flux. The modulation frequency range of our HF-MPL setup has been extended to $200 \mathrm{MHz}$ to investigate fast carriers' dynamics while keeping high sensitivity detection, similarly to TRPL techniques. The 
fittings on the experimental data of both TRPL and MPL experiments were performed by using a drift diffusion simulator developed in our institute to investigate the surface recombination mechanism ${ }^{19}$. Two other strong points of our approach are: i) The simultaneous fit of data acquired from the two technics and for different illumination levels; ii) the correction of the MPL and TRPL data with respects to the system optical transmission rather than a normalization to unity. These two features strongly reduce the risk of finding an unphysical solution when fitting the data with numerous parameters. This parallel experimental and modelling approach goes beyond a simple TRPL data analysis, it evidences a quantitative SRV measurement as well as the determination of minority carrier lifetime, defect density, capture cross section for both electrons and holes and defect levels ${ }^{27}$. It is a continuation of the methodology of Hasegawa's group extending it to time resolved technics. In the next section we describe the samples and the methodology, then the results will be presented and discussed.

\section{METHOD}

\section{Sample description}

The InP $350 \mu \mathrm{m}$ thick samples are Sn doped with an n-type doping level was measured by Mott-Schottky method to be around $3 \times 10^{18} \mathrm{~cm}^{-3}$. The four samples are studied coming from the same wafer with different surface treatments presented in the Table 1. Poly-phosphazen layer was deposited on "PH" sample in liquid ammonia to form a very thin film which has been characterised by XPS experiment. XPS data for the film are inserted in supplementary material showing the strong uniformity and small thickness of the film. The charge current monitoring during the deposition process gives a one monolayer of phosphazen formation which is consistent with XPS data. ${ }^{26}$. "Epi" sample (epi-ready wafer), was left untreated from supplier. "Desox" has been cleaned by $2 \mathrm{M} \mathrm{HCl}$ solution before the measurement in order to eliminate the oxide layer. After two weeks in ambient atmosphere, we measured "Desox" again as "Reox" 
Table 1 Samples' basic information for experimental measurements and simulations ${ }^{28}$

\begin{tabular}{|c|c|c|c|c|}
\hline Sample & Epi & Desox & Reox & $\mathrm{PH}$ \\
\hline $\mathrm{HCl}$ surface cleaning (2 M) & No & Yes & $\begin{array}{c}\text { Yes } \\
\text { (2weeks in } \\
\text { air after) }\end{array}$ & No \\
\hline Poly-phosphazen layer & No & No & No & Yes \\
\hline Doping density $\left(\mathrm{cm}^{-3}\right)$ & \multicolumn{4}{|c|}{$3 \times 10^{18}$} \\
\hline Thickness $(\mu \mathrm{m})$ & \multicolumn{4}{|c|}{350} \\
\hline Bandgap, $E_{g}(e V)$ & \multicolumn{4}{|c|}{1.30} \\
\hline Relative permittivity, $\varepsilon_{\mathrm{r}}$ & \multicolumn{4}{|c|}{12.5} \\
\hline Electron affinity (eV) & \multicolumn{4}{|c|}{4.38} \\
\hline Electron mobility, $\mu_{\mathrm{n}}\left(\mathrm{cm}^{2} \mathrm{~V}^{-1} \mathrm{~s}^{-1}\right)$ & \multicolumn{4}{|c|}{720} \\
\hline Hole mobility, $\mu_{\mathrm{p}}\left(\mathrm{cm}^{2} \mathrm{~V}^{-1} \mathrm{~s}^{-1}\right)$ & \multicolumn{4}{|c|}{80} \\
\hline Electron thermal velocity, $v_{\mathrm{n}}\left(\mathrm{cm} \cdot \mathrm{s}^{-1}\right)$ & \multicolumn{4}{|c|}{$3.9 \times 10^{7}$} \\
\hline Hole thermal velocity, $v_{\mathrm{p}}\left(\mathrm{cm} \cdot \mathrm{s}^{-1}\right)$ & \multicolumn{4}{|c|}{$1.7 \times 10^{7}$} \\
\hline Conduction and Valence band density of & \multicolumn{4}{|c|}{$5.7 \times 10^{17}$} \\
\hline states, $\mathrm{N}_{\mathrm{c}}, \mathrm{N}_{\mathrm{v}}\left(\mathrm{cm}^{-3}\right)$ & \multicolumn{4}{|c|}{$1.1 \times 10^{19}$} \\
\hline
\end{tabular}

\section{Measurement principle}

a)

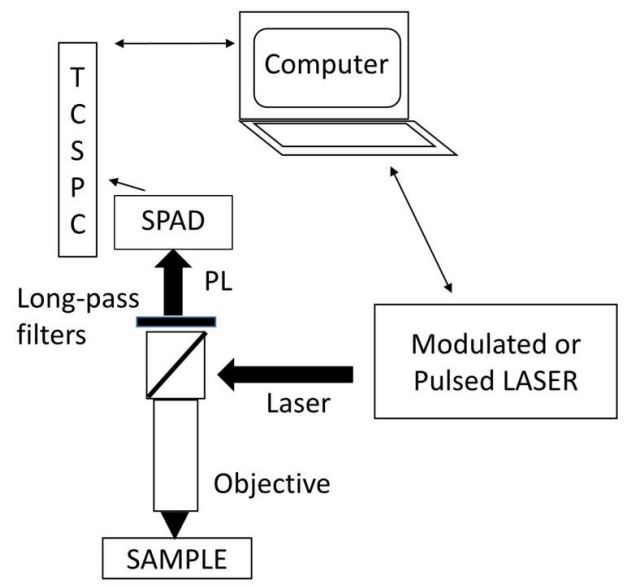

b)

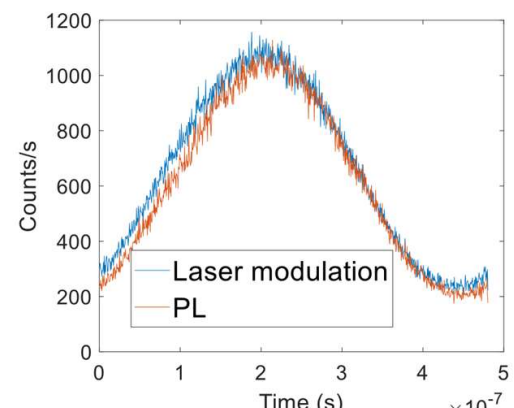

c)

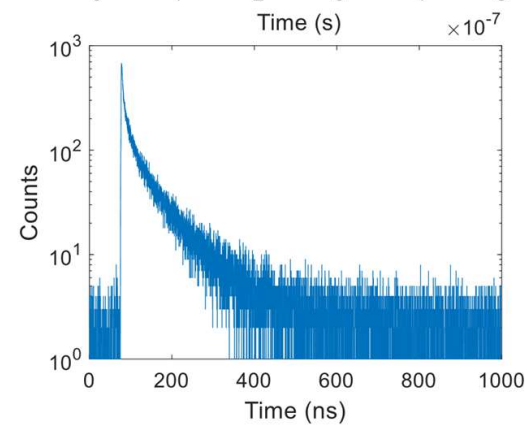

Figure 1 (a) Sketch of the MPL/TRPL experimental setup. The setup is a conventional microscope using long pass filters for separating the laser light and the photoluminescence; (b) Modulated signals: direct measurement of the laser light (blue) when removing the long pass filters, PL signal of an InP wafer sample (red) presenting a phase shift (delay) with respects to the laser light modulation; (c) Typical TRPL decay. 
The setup is sketched in Figure 1. The light sources are a $638 \mathrm{~nm}$ wavelength intensity modulated laser and a $532 \mathrm{~nm}$ pulsed laser with 80 ps pulse duration at $5 \mathrm{MHz}$ repetition rate. The modulation is based on sinusoidal variations of the light intensity around a given working point. In this study the ratio between modulated and continuous part of the modulated light is close to $60 \%$. Samples are excited with a beam diameter of $50 \mu \mathrm{m}$ : it ensures an excitation spot larger than the diffusion length avoiding lateral transport of the photogenerated carriers which might influence the carrier dynamics. To detect the PL signal with an optimal sensitivity we used a Single Photon Avalanche Detector. Then, the Picoharp 300 Time Correlated Single Photon Counter (TCSPC) and the in house LabVIEW code allow reconstructing one decay in TRPL mode or one period of the modulated signal. In such configuration we obtain a unique experimental setup to record fast modulated signal with a high detection sensitivity, contrary to usual modulated experiments where the sensitivity is strongly degraded because of the use of fast detector (compromise between speed and gain). Once the modulated signal is recorded, we can extract the phase and amplitude of the first harmonic by fast Fourier transform. Finally, we record the phase and the amplitude of the first harmonic at each frequency and we calculate the phase shift between the laser and photoluminescence.

\section{Simulation procedure}

The fitting procedure was performed by using a in house LabVIEW simulator based on drift-diffusion equations, the Shockley Read Hall (SRH) recombination model and optical models 19. The fits were made using a least square minimization procedure with normalized error (see Supplementary Materials). The absorption coefficient was measured and taken into account (see Supplementary Material). Details of the simulation are given just below. Please note that for each sample, the fit was performed simultaneously to all the TRPL decays and the MPL real and imaginary parts leading to a unique set of parameters. Furthermore, the TRPL and MPL maximal intensities were not normalized to unity as in usual procedures. At high laser fluence, we used neutral densities to avoid detector saturation by the emitted PL. We measured the attenuation of the densities at the PL wavelength and corrected the data by dividing by these attenuation factors. 
The experimental data were obtained under several illumination flux and fluences. We note that the samples are illuminated until a steady transient emission is achieved. The MPL phase and amplitude spectra were depicted by Fourier transform for modulated frequency ranging from 50 $\mathrm{kHz}$ to $200 \mathrm{MHz}$ and fluxes varying from $5 \times 10^{14}$ to $3 \times 10^{21}$ photons. $\mathrm{cm}^{-2} \mathrm{~s}^{-1}$ (equivalent for about 0.005 sun to 30000 sun). The TRPL decays were recorded at the same sample location by different excitation powers with illumination fluences from $7 \times 10^{6}$ photons.pulse $e^{-1} \cdot \mathrm{cm}^{-2}$ to $9 \times 10^{13}$ photons.pulse ${ }^{-1} \cdot \mathrm{cm}^{-2}$

\subsection{Bulk Simulation}

The one-dimensional transport of electrons and holes in a semiconductor material can be efficiently represented by the drift-diffusion system (equations 1-4) coupled to a Poisson equation (5). With the electron and hole densities (n, p), the respective currents (Jn,p), and the diffusion coefficients $\left(D_{n, p}\right)$ and mobilities $\left(\mu^{n, p}\right)$, the optical generation rate $(G)$, the recombination rate $\left(\mathrm{R}_{\mathrm{rec}}\right)$, the electric field $(\mathrm{F})$ and the doping level $\left(\mathrm{C}_{\mathrm{dop}}\right)$, the equation system reads:

$$
\begin{gathered}
\frac{\partial n}{\partial t}=\frac{1}{q} \operatorname{div}\left(J_{n}\right)+G-R_{r e c} \\
\frac{\partial p}{\partial t}=-\frac{1}{q} \operatorname{div}\left(J_{p}\right)+G-R_{r e c} \\
J_{n}=D_{n} \operatorname{grad}(n)+\mu_{n} n F \\
J_{p}=D_{p} \operatorname{grad}(p)-\mu_{p} p F \\
\operatorname{div}(F)=\frac{q}{\varepsilon}\left(p-n+C_{d o p}\right)
\end{gathered}
$$


The two first equations express the charge conservation as a function of the currents, the generation, the recombination. The currents of electrons and holes are separated and associated to diffusion and drift terms (3-4). The Poisson equation completes the system (5). The onedimensional drift diffusion simulation code allows us to reproduce the HF-MPL/TRPL data at the same time with the same parameters. The code uses a 1D spatial discretization of the drift diffusion equations as presented in ${ }^{29,30}$ in combination with Differential Algebraic Equations solvers (DAEs) for the temporal part. MATLAB ODE15s was used but IDAS ${ }^{31}$ can also be used with similar results. This approach has been proved to be efficient ${ }^{32}$. The code includes the following recombination mechanisms: radiative, Auger, $\mathrm{SRH}^{1}$. The outputs provide the carriers densities profiles $n$ and $p$ as well as the electric potential for a given semiconductor stack with respect to depth and time. Light excitation $\mathrm{G}(\mathrm{t}, \mathrm{x})$ can follow arbitrary time profiles, such as short impulsion (TRPL) or sinusoidal variations (MPL).

Since the doping level is high $\left(3 \times 10^{18} \mathrm{~cm}^{-3}\right)$ for all samples, and the injection level during TRPL never reaches the doping level for these samples, we chose to represent the recombination in the bulk by a single lifetime $\tau_{\mathrm{b}}$. Then with $\mathrm{n}_{\mathrm{i}}$ being the intrinsic concentration,

$$
R_{r e c}=\frac{n p-n_{i}^{2}}{n \tau_{b}}
$$

The boundary condition at the backside involves local electro neutrality, which is a good assumption since the photo generated carriers do not reach the back side. At the front side, we use the SRH formalism for the boundary conditions.

\footnotetext{
${ }^{1} \mathrm{SRH}$ can use the stationary or time dependent form, i.e rate equations. In the second case a supplementary equation is added governing the trap filling. The charge of the trap, donor-like or acceptor like, is taken into account
} 


\subsection{Shockley Read Hall surface time dependent recombination}

The recombination at silicon surface was already successfully represented by Aberle ${ }^{33}$ using SRH formalism in stationary regime. Rosenwaks also took into account the surface charge created by the difference of capture rates for electrons and holes at the surface in time dependent experiments ${ }^{34}$. A common approximation in the literature is to use stationary SRH formula even in the case of transient experiments, but it. can lead to erroneous results, so it was not used. by representing the recombination at the surface or at the InP surface or at the $\mathrm{InP} /$ poly-phosphazen interface by a time dependent SRH mechanism. It involves a continuous energetic distribution of traps at its surface. For each energy level of the distribution, assuming that the density of traps per surface unit and per energy unit is $N_{T}(E)$ and the corresponding number of trapped electrons $\mathrm{n}_{\mathrm{T}}(\mathrm{E})$, the emission and capture rates of the surface levels lying inside the bandgap for both type of carriers are classically expressed in Shockley Read Hall theory as follow ${ }^{35}$ :

$$
\begin{gathered}
\text { Emission }_{n}=\sigma_{n} v_{n} n^{*} n_{T}(E) \\
\text { Capture }_{n}=\sigma_{n} v_{n} n\left(N_{T}(E)-n_{T}(E)\right) \\
\text { Emission }_{p}=\sigma_{p} v_{p} p^{*}\left(N_{T}(E)-n_{T}(E)\right) \\
\text { Capture }_{p}=\sigma_{p} v_{p} p n_{T}
\end{gathered}
$$

Here $\sigma_{\mathrm{n}, \mathrm{p}}$ stands for the capture cross sections of the defects for electrons and holes, respectively, $v_{\mathrm{n}, \mathrm{p}}$ stands for the thermal velocities for electrons and holes, respectively, $\mathrm{Nt}(\mathrm{E})$ is the trap density per surface unit are and nt is the density of trapped electrons per surface unit.

These emission and capture rates represent the capture and emission rate $\left(\mathrm{cm}^{-3} \cdot \mathrm{s}^{-1}\right)$ for electrons of the conduction band at the surface $((7)$ and (8)) and for holes from the valence band ((9) and (10)) for each energetic level present in the surface density of states. One can note that the capture rates involve bimolecular processes $\left(n *(\mathrm{Nt}-\mathrm{nt})\right.$ product or $\mathrm{p}^{*} \mathrm{nt}$ product) where the emission rates are monomolecular and involve $\mathrm{n}^{*}$ and $\mathrm{p}^{*}$ quantities. The quantities $\mathrm{p}^{*}$ and $\mathrm{n}^{*}$ are related to the 
conduction and valence band equivalent densities of states $\mathrm{Nc}, \mathrm{Nv}$ and the corresponding band gap edges Ec, Ev:

$$
\begin{aligned}
& n^{*}=N_{c} \exp \left(-\frac{E c-E}{k T}\right) \\
& p^{*}=N_{v} \exp \left(-\frac{E-E v}{k T}\right)
\end{aligned}
$$

And the system is determined by the set of time dependent equations:

At each $\mathrm{E}$,

$$
\begin{gathered}
\frac{\partial n_{T}(E)}{\partial t}=\text { Capture }_{n}-\text { Emission }_{n}+\text { Emission }_{p}-\text { Capture }_{p} \\
\frac{\partial J_{\text {sur }_{n}}}{\partial E}=-q\left(\text { Captur }_{n}-\text { Emission }_{n}\right) \\
\frac{\partial J_{\text {sur }_{p}}}{\partial E}=q\left(\text { Capture }_{p}-\text { Emission }_{p}\right)
\end{gathered}
$$

Finally, the total charge at the surface for donor-like and acceptor-like traps as well as the total electrons and holes currents will be, with q the positive elementary charge:

$$
\begin{gathered}
Q_{\text {trap acceptor }}=-q \int_{E_{v}}^{E_{c}} n_{T}(E) d E \\
Q_{\text {trap donor }}=q \int_{E_{v}}^{E_{c}}\left(N_{T(E)}-n_{T}(E)\right) d E \\
J_{\text {surf }_{n}}=\int_{E_{v}}^{E_{c}} \frac{\partial J_{\text {surf }}}{\partial E} d E
\end{gathered}
$$




$$
J_{\text {sur } p}=\int_{E_{v}}^{E_{c}} \frac{\partial J_{\text {surf }}}{\partial E} d E
$$

The electric field at the surface will follow:

$$
\varepsilon F_{\text {surf }}=\text { Charge }_{\text {trap }}
$$

The above formula represents the currents and the charge changes for donor or acceptor defects at the surface. In the case of stationary regime, we get the following currents and charge at the surface ${ }^{33}$.

$$
\begin{gathered}
J_{\text {surf } n_{n}}=J_{\text {surf }_{p}}=q \int_{E_{v}}^{E_{c}} \frac{n_{s} p_{s}-n_{i}^{2}}{\frac{n_{s}+n^{*}}{S_{p}}+\frac{p_{s}+p^{*}}{S_{n}}} d E \\
Q=q \int_{E_{v}}^{E_{c}} \frac{N_{T}\left(\sigma_{n} n+\sigma_{p} p^{*}\right)}{\sigma_{n}\left(n+n^{*}\right)+\sigma_{p}\left(p+p^{*}\right)} d E \\
Q_{\text {trap donor }}=q \int_{E_{v}}^{E_{c}} \frac{N_{T}\left(\sigma_{n} n^{*}+\sigma_{p} p\right)}{\sigma_{n}\left(n+n^{*}\right)+\sigma_{p}\left(p+p^{*}\right)} d E
\end{gathered}
$$

$S_{n}$ and $S_{p}$ are so called surface recombination velocities and are given by

$$
\begin{aligned}
& S_{n}=N_{T} \sigma_{n} v_{n} \\
& S_{p}=N_{T} \sigma_{p} v_{p}
\end{aligned}
$$

The analysis made in this work will be performed with a single level and with the time dependent equations but the values of the stationary SRV will be given for comparison with literature. 


\section{RESULTS AND DISCUSSION}

Using the simplest model where only the bulk lifetime of minority carriers and minority carrier SRV were simulated as fitting parameters. The fitting results were not satisfactory because using a single SRV is not sufficient to reproduce the injection dependence of the signal. For similar samples (see Supplementary Material) simulation gave an excessively long bulk lifetime of $42 \mathrm{ns,}$ higher than the lifetime of radiative recombination which is $15 \mathrm{~ns}$ (maximum value calculated from the literature : $k_{\text {rad }}$ ranges from $2 \times 10^{-11}$ to $1.2 \times 10^{-10} \mathrm{~cm}^{3} \cdot \mathrm{s}^{-1} 36,37$ ).

We therefore had to upgrade the model up to a sufficient complexity with five parameters including minority carrier lifetime in bulk $\left(\boldsymbol{\tau}_{\text {bulk }}\right)$, defect density $\left(N_{t}\right)$, capture cross section for both electrons $\left(\sigma_{n}\right)$ and holes $\left(\sigma_{p}\right)$ and defect level $\left(\boldsymbol{E}_{t}\right)$. Note that we choose to introduce one single level instead of a continuous distribution. It assumes that the surface recombination rates can be represented by with only four parameters. We will discuss the validity of this assumption later.

Fitting parameters are summarized in table 2. A range is given rather corresponding to the $95 \%$ confidence interval (except for the surface recombination velocities extracted from the fit). Once the fitting parameters were extracted, the maximal excess carrier density for each illumination flux has been calculated by equations (1)-(20) and plotted in Figure 2. According to our solver, the hole quasi-Fermi level position is found at $1.27 \mathrm{eV}$ from conduction band for TRPL and $1.22 \mathrm{eV}$ for MPL at the maximum illumination, meaning that the surface defect system is scanned nearly all over the bandgap (see band diagram in the Supplementary Material).

It can be observed that the maximal excess carrier densities are linearly dependent with the illumination fluences for at low illumination and deviate slightly at high fluences. However, the injection level remains below the doping level.

Theoretically, the comparison of the MPL experimental and calculated average intensity at high illumination fluxes could allow to perform the Photoluminescence Surface State Spectroscopy method ${ }^{14,15}$. Unfortunately, at such high doping level it would require more than $10^{22}$ photons.cm ${ }^{2} \cdot \mathrm{s}^{-1}$ and it would become difficult to prevent sample heating and Auger recombination. 
For all samples, the bulk lifetime was determined to be in the range 10-13 ns. Regarding the high doping level, it implies that the radiative constant is in the same order of magnitude than the one found by Liu and Rosenwaks ${ }^{16}$ which report the lowest value in the literature $\left(2 \times 10^{-11} \mathrm{~cm}^{-}\right.$ $\left.{ }^{3} \cdot \mathrm{s}^{-1}\right)$. Any larger value would shorten the decays and reduce the MPL phase.
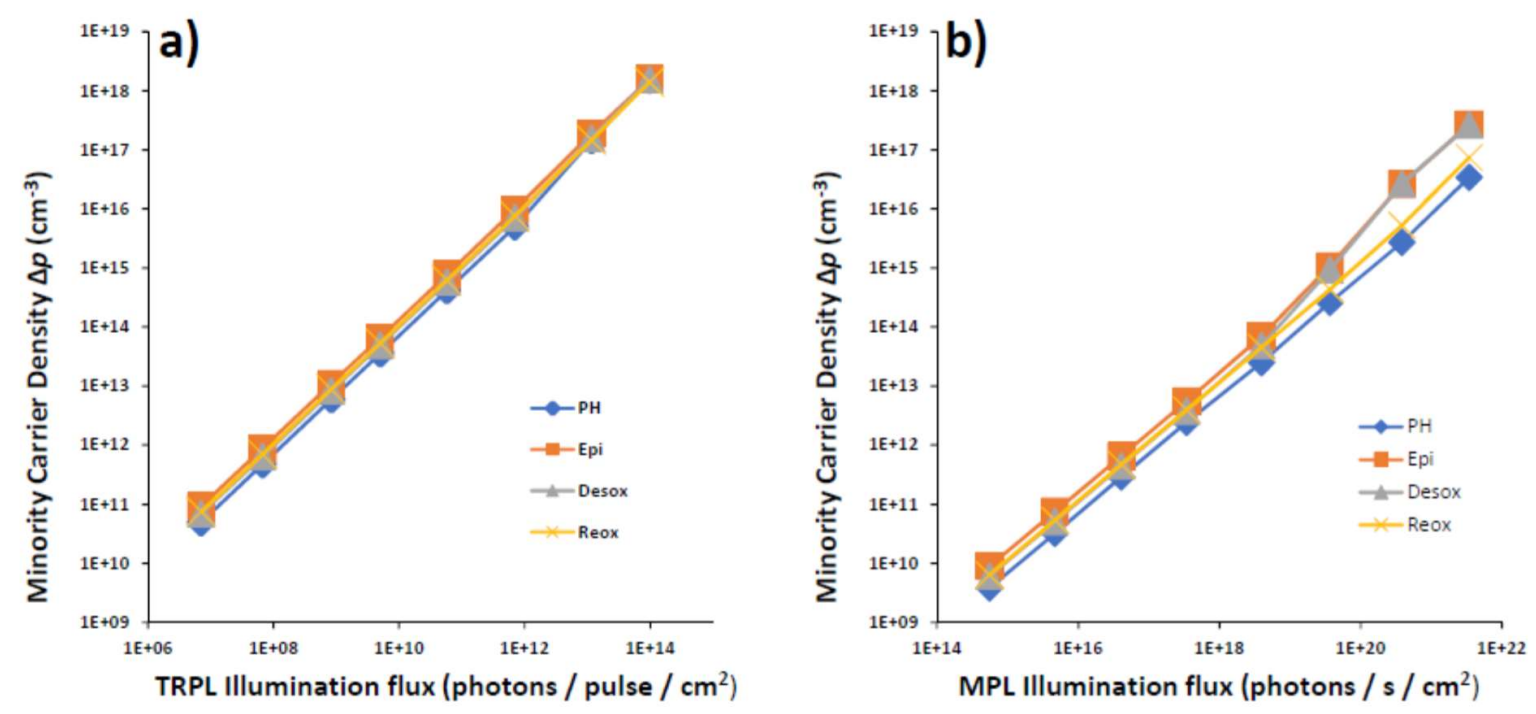

Figure 2 Simulated maximal injection level presented by excess carrier density vs illumination power for a) TRPL and b) MPL for the four samples

Table 2 Detailed fitting results for conjugated MPL/TRPL simultaneous fitting with surface defect on the four samples

\begin{tabular}{c|cccc}
\hline Fitting Parameters & PH & Epi & Desox & Reox \\
\hline Bulk lifetime, $\tau_{\text {bulk }}(\mathrm{ns})$ & \multicolumn{4}{|c}{$12(10-13)$} \\
\hline $\begin{array}{c}\text { Defect density, } N_{t}\left(10^{10}\right. \\
\left.\mathrm{cm}^{-2}\right)\end{array}$ & 20 & 4 & 60 & 360 \\
\hline $\begin{array}{c}\text { Electron capture cross- } \\
\text { section, } \sigma_{n}\left(\mathrm{~cm}^{2}\right)\end{array}$ & $\begin{array}{c}2 \times 10^{-16} \\
\left(4-100 \times 10^{-17}\right)\end{array}$ & $\begin{array}{c}\left(0.7-2 \times 10^{-17}\right) \\
(4-8)\end{array}$ & $\left(4-8 \times 10^{-19}\right)$ & $\left(2-6 \times 10^{-17}\right)$ \\
\hline $\begin{array}{c}\text { Hole capture cross-section, } \\
\sigma_{p}\left(\mathrm{~cm}^{2}\right)\end{array}$ & $4 \times 10^{-13}$ & $8 \times 10^{-14}$ & $1 \times 10^{-14}$ & $6 \times 10^{-16}$ \\
$\left(1-18 \times 10^{-13}\right)$ & $4-15 \times 10^{-14}$ & $\left(0.8-2 \times 10^{-14}\right)$ & $\left(0.3-1 \times 10^{-15}\right)$ \\
\hline
\end{tabular}




\begin{tabular}{c|cccc}
\hline $\begin{array}{c}\text { Surface Recombination } \\
\text { Velocity, } S_{n}=N_{t} \sigma_{n} v_{n} \\
\left(\mathrm{~cm} \cdot \mathrm{s}^{-1}\right)\end{array}$ & $\mathbf{2 \times 1 0 ^ { 3 }}$ & $\mathbf{2 4}$ & $\mathbf{1 2}$ & $\mathbf{6 \times 1 0 ^ { 3 }}$ \\
\hline $\begin{array}{c}\text { Surface Recombination } \\
\text { Velocity, } S_{p}=N_{t} \sigma_{p} v_{p} \\
\left(\mathrm{~cm} . \mathrm{s}^{-1}\right)\end{array}$ & $\mathbf{1 \times 1 0 ^ { 6 }}$ & $\mathbf{5 \times 1 0 ^ { 4 }}$ & $\mathbf{1 \times 1 0 ^ { 5 }}$ & $\mathbf{4 . 5} \times \mathbf{1 0}^{\mathbf{3}}$ \\
\hline
\end{tabular}

\section{Sample comparison}

The data of the PH sample are presented in figure 3 for different excitation intensities. Other data are presented in the Supplementary Material. The specificity of the PH sample is that the temporal response remains similar over eight orders of magnitude (except the background at low intensity curves). This is due in part to slightly larger capture cross sections for both electrons and holes of the defects system in compare to the three other samples. This enhances surface recombination and prevents surface trap saturation. For such highly doped samples, the surface has a higher recombination rate than the other samples and the poly-phosphazen is not found to reduce the surface recombination.

All the other samples present an injection dependence on both the MPL and the TRPL signature. This dependence is mainly due to the asymmetric capture cross sections, the postulated trap center being not able to capture enough electrons at high fluences. The fits were however not completely satisfactory at really high fluence (two last TRPL decays). This can be due to measurement uncertainty, but also to the appearance of another mechanism such like radiative or Auger recombination. We can also note that the fit of the MPL phase is unsatisfactory for "Desox", while the amplitude and the TRPL decays are well fitted. Indeed, it raised a question about the chemical stability of 'Desox' surface during the experiment. As the holes are strong reducing species, a photo induced re-oxygenation of the surface at strong illumination fluxes may be an explanation for this behaviour.

In comparison to references ${ }^{14,38}$, we found similar magnitude for the hole capture cross sections when our electron capture cross section are smaller by several order of magnitude. We found these capture cross sections to be sample dependent. As different capture cross sections would strongly change the PL intensity dependence to illumination fluxes and fluences for either MPL or TRPL 
or both, we conclude that the samples surface are different from ${ }^{38}$. The calculated minority carrier SRV are also in the agreement with those reported in the literature, where for the doping density from $3 \times 10^{15} \mathrm{~cm}^{-3}$ to $3 \times 10^{18} \mathrm{~cm}^{-3}$ caused an increase of SRV from $5000 \mathrm{~cm} / \mathrm{s}$ to $1 \times 10^{6} \mathrm{~cm} / \mathrm{s}{ }^{9}$. Note we also provided the majority carrier SRV which allows for a real simulation of the surface.
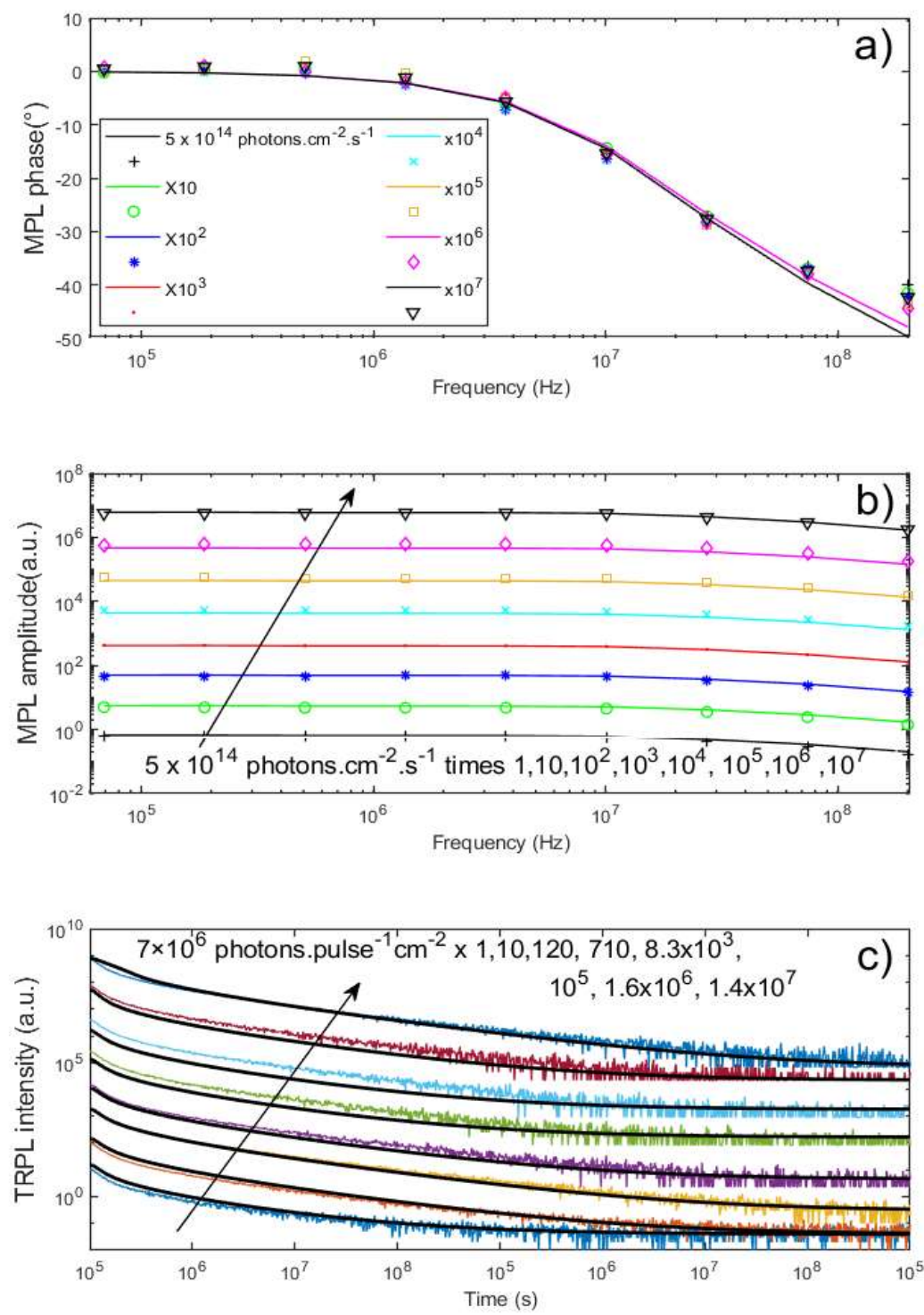
Figure 3 Conjugated MPL/TRPL simultaneous fitting with surface defect on PH sample. MPL phase (a), amplitude (b). Experimental data are in symbols and fitting curves are in line; TRPL decays (c) and fits. Detailed fitting results are presented in Table 2.

3.Discussions about the oxide charge, and the surface density of state.

Surface photovoltage experiments (SPV) were performed indicating no significant band bending at the samples surface for PH, 'Reox' and 'Epi' sample. According to our simulations, an additional charge superior to $10^{12}$ elementary charges per $\mathrm{cm}^{-2}$ is necessary to modify the TRPL and MPL response. Indeed, our fitting procedure always converged to fixed charges inferior to this value. A higher charge was found to be incompatible with the observed injection dependence of the data

In the present article we chose to represent the surface density of state by a single level. This could correspond to three different cases. First case involves a real single level at the surface due for example to an impurity (most probably the case of the sample "EG1" presented in Supplementary Material). In the second case the surface density of states (DOS) is dominated by deep centers. In this case the reemission terms are negligible due to the smallness of $n^{*}$ and $p^{*}$ and the postulated single level represent the total density of state of these levels, as the full distribution is not experimentally accessible. In the third case, which is the most probable, the model is correctly simulating a complex density of state at the surface.

We also simulated a U-shaped density of states for the two samples 'Desox' and 'Reox' in accordance with the DIGS model ${ }^{13-15}$ (see Supplementary Material). This kind of shape has often been found to be present on oxidized surfaces by capacitance measurements. The electroneutrality level was taken in the literature as it supposed to be pinned for a given material in the DIGS model. We were able to reproduce the fits with a slightly larger fitting error using the same capture cross sections for both 'Reox' and 'Desox' sample, two samples where there is a probable disordered layer at the surface due to reoxidation. Knowing this fact, our model has the advantage of reducing the number of fitting parameters, the recombination rates at the surface being correctly modelled over eight orders of magnitude of illumination. Extracted DOS are presented in Supplementary Materials. 


\section{CONCLUSIONS}

We have developed a combined characterization approach to reveal carriers trapping mechanism, by using on the same sample two powerful techniques: TRPL and MPL. This combined approach provides complementary information on carrier recombination and passivation effect of InP by poly-phosphazen was studied. Four different samples were investigated: $\mathrm{n}$ type highly doped bare surface InP in three oxidation states, and $n$ type highly doped passivated InP. We established a modelling approach from a drift-diffusion model which includes different defect recombination mechanisms including surface SRH recombination center with defect density, capture cross sections for both electrons and holes and defect level and radiative recombination coefficient. The injection level study indicates a monomolecular recombination of minority hole carriers in the bulk. The recombination rates of the surface were successfully characterized by the model, when the real DOS of is not accessible without strong assumptions. The minority carrier SRVs were calculated from the trap densities and capture cross-sections, varying from $10^{4}$ to $10^{6}$ $\mathrm{cm} / \mathrm{s}$ for different InP surfaces.

The poly-phosphazen is found to modify the recombination kinetic, increasing the recombination rate by an increase of the capture cross sections, the density of defect remaining similar. We also highlighted that the poly-phosphazen allowed a similar time response over eight decades of minority carrier density. An electronic fast switch or optical fast modulated laser would provide the great advantage of an invariant time response over eight orders of magnitude of the minority carrier density and of the chemical stability of the surface at high fluences.

Alternatively, a chemical photo-modification of the surface at high illumination fluxes would be a hypothesis to investigate for the other samples, especially for the clean surface.

\section{SUPPLEMENTARY MATERIALS}

The Supplementary Materials will present respectively the XPS data, the first fitting trial with a two-parameter model, the model for calculating the photoluminescence and extracting the 
absorption coefficient, the fitting error calculation, the remaining figures for the last three samples, the results using the Unified Disorder Induced Gap State model.

\section{AKNOWLEDGMENTS}

This work was supported by the French government in the frame of the project EPINAL (Projet-ANR17-CE08-0034) and by the program of investments for the future (Programme d'Investissement d'Avenir ANR-IEED-002-01) in the frame of program IV ("advanced characterization") of the Institut Photovoltaïque d'lle de France. We gratefully acknowledge Albert Cohen and Matthieu Dolbeault from Jacques-Louis Lions Laboratory (LLL) for their support on least square procedures, and James Connolly for proofreading the final version of the article and for interesting discussions.

\section{DATA AVALAIBILITY STATEMENT}

The data that support the findings of this study are available from the corresponding author upon reasonable request.

\section{REFERENCES}

${ }^{1}$ G. Purvis, III-Vs Review 17, 35 (2004).

${ }^{2}$ M. Smit, K. Williams, and J. van der Tol, APL Photonics 4, 050901 (2019).

${ }^{3}$ L.E. Black, A. Cavalli, M.A. Verheijen, J.E.M. Haverkort, E.P.A.M. Bakkers, and W.M.M. Kessels, Nano Lett. 17, 6287 (2017).

${ }^{4}$ Y. Tao, A. Yelon, E. Sacher, Z.H. Lu, and M.J. Graham, Appl. Phys. Lett. 60, 2669 (1992).

${ }^{5}$ Y. Lin, R. Kapadia, J. Yang, M. Zheng, K. Chen, M. Hettick, X. Yin, C. Battaglia, I.D. Sharp, J.W. Ager, and A. Javey, J. Phys. Chem. C 119, 2308 (2015).

${ }^{6}$ S.D. Stranks, V.M. Burlakov, T. Leijtens, J.M. Ball, A. Goriely, and H.J. Snaith, Physical Review Applied 2, 1 (2014).

${ }^{7}$ D. Huppert and Y. Rosenwaks, Physical Review B 44, 97 (1991).

${ }^{8}$ Y. Rosenwaks, Y. Shapira, and D. Huppert, Applied Physics Letters 57, 2552 (1990).

${ }^{9}$ S. Bothra, S. Tyagi, S.K. Ghandhi, and J.M. Borrego, Solid State Electronics 34, 47 (1991).

${ }^{10}$ J.M. Moison, M. Van Rompay, and M. Bensoussan, Applied Physics Letters 48, 1362 (1986).

${ }^{11}$ J.M. Moison and M. Bensoussan, Revue de Physique Appliquée 22, 293 (1987). 
12 C.A. Hoffman, K. Jarašiūnas, H.J. Gerritsen, and A. V. Nurmikko, Appl. Phys. Lett 33, 536 (1978).

${ }^{13}$ H. Hasegawa, J. Vac. Sci. Technol. B 4, 1130 (1986).

${ }^{14}$ M. Miczek, B. Adamowicz, and H. Hasegawa, Surface Science 507-510, 240 (2002).

15 P. Tomkiewicz, B. Adamowicz, M. Miczek, H. Hasegawa, and J. Szuber, Applied Surface Science 254, 8046 (2008).

${ }^{16}$ A. Liu and Y. Rosenwaks, Journal of Applied Physics 86, 430 (1999).

17 W. Favre, J.-P. Kleider, D. Muñoz, S. Martin-de-Nicolás, and P.-J. Ribeyron, Physica Status Solidi (c) 8, 775 (2011).

18 B. Bérenguier, N. Barreau, A. Jaffre, D. Ory, J.F. Guillemoles, J.-P. Kleider, and L. Lombez, Thin Solid Films 669, 520 (2018).

${ }^{19}$ B. Berenguier, N. Moron, W. Zhao, J.F. Guillemoles, J.-P. Kleider, and L. Lombez, in IEEE 46th Photovoltaic Specialists Conference (PVSC),Chicago, IL, USA (2019), p. 352-358.

${ }^{20}$ K.B. Choi, C.S.P. Teena, J.W. Ho, J. Wang, J. Lin, A.A. Bettiol, A.G. Aberle, and J. Wong, IEEE Journal of Photovoltaics 7, 1687 (2017).

${ }^{21}$ S. Miasojedovas, P. Vitta, I. Reklaitis, R. Kudz, and I. Pietzonka, 45, 3290 (2016).

22 R. Tomašiūnas, A. Hoffmann, I. Reklaitis, F. Nippert, and R. Kud, 035701, (2017).

${ }^{23}$ A. Etcheberry, A.-M. Goncalves, C. Mathieu, J. Vigneron, N. MEZAILLES, and P.L. FLOCH, US20150108617A1 (23 April 2015).

${ }^{24}$ A.-M. Gonçalves, N. Mézailles, C. Mathieu, P. Le Floch, and A. Etcheberry, Chem. Mater. 22, 3114 (2010).

25 A.-M. Gonçalves, O. Seitz, C. Mathieu, M. Herlem, and A. Etcheberry, Electrochemistry Communications 10, 225 (2008).

26 A.-M. Gonçalves, C. Njel, D. Aureau, and A. Etcheberry, Applied Surface Science 391, 44 (2017).

${ }^{27}$ W. Zhao, C. Rakotoarimanana, A.M. Goncalves, A. Etcheberry, L. Lombez, B. Bérenguier, and J.-F. Guillemoles, 3 (n.d.).

${ }^{28}$ (n.d.).

${ }^{29}$ P. Farrell, N. Rotundo, D. Hai Doan, M. Kantner, J. Fuhrmann, and T. Koprucki, Numerical Methods for Drift-Diffusion Models (Weierstraß-Institut für Angewandte Analysis und Stochastik, 2016).

${ }^{30} \mathrm{~S}$. Ibrahim, Modélisation et Caractérisation Des Cellules Photovoltaïques à Haut Rendement à Base d'hétérojonctions Combinant Silicium Cristallin et Couches Minces Polymorphes, Paris 11, 2010.

31 A.C. Hindmarsh, P.N. Brown, K.E. Grant, S.L. Lee, R. Serban, D.E. Shumaker, and C.S. Woodward, ACM Transactions on Mathematical Software (TOMS) 31, 363 (2005). 
${ }^{32}$ C. de Falco, R. Sacco, and M. Verri, Computer Methods in Applied Mechanics and Engineering 199, 1722 (2010).

${ }^{33}$ A.G. Aberle, Crystalline Silicon Solar Cells: Advanced Surface Passivation and Analysis (Centre for Photovoltaic Engineering, University of New South Wales, 1999).

${ }^{34}$ Y. Rosenwaks, B.R. Thacker, R.K. Ahrenkiel, A.J. Nozik, and I. Yavneh, Physical Review B 50, 1746 (1994).

${ }^{35}$ W. Shockley and W.T. Read, Physical Review 87, 835 (1952).

${ }^{36}$ O. Semyonov, A. Subashiev, Z. Chen, and S. Luryi, Journal of Applied Physics 108, 013101 (2010).

${ }^{37}$ I. Tsimberova, Y. Rosenwaks, and M. Molotskii, 93, 9797 (2003).

${ }^{38}$ M. Miczek, B. Adamowicz, J. Szuber, and H. Hasegawa, Vacuum 63, 223 (2001). 